\title{
Study of vector meson fragmentation using a broken SU(3) model
}

\author{
D. Indumathi \\ Institute of Mathematical Sciences, \\ CIT Campus, \\ Chennai 600 113, India \\ indu@imsc.res.in \\ H. Saveetha \\ Department of Theoretical Physics, \\ University of Madras, \\ Guindy Campus, Chennai 600 025, India. \\ saveehari@gmail.com
}

\begin{abstract}
Inclusive hadro production in $e^{+} e^{-}$annihilation processes is examined to study the fragmentation process. A broken $\mathrm{SU}(3)$ model is used to determine the quark and gluon fragmentation functions of octet vector mesons, $\rho$ and $K^{*}$, in a simple way with an $\mathrm{SU}(3)$ breaking parameter $\lambda$. These are expressed in terms of just two light quark fragmentation functions, $V\left(x, Q^{2}\right)$ and $\gamma\left(x, Q^{2}\right)$ and the gluon fragmentation function $D_{g}\left(x, Q^{2}\right)$. These functions are parameterized at the low input scale of $Q_{0}^{2}=1.5 \mathrm{GeV}^{2}$, evolved through LO DGLAP evolution including charm and bottom flavour at appropriate thresholds, and fitted by comparison with data at the $Z$-pole. The model is extended with the introduction of a few additional parameters to include a study of singlet-octet mixing and hence $\omega$ and $\phi$ fragmentation. The model gives good fits to the available data for $x \gtrsim 0.01$, where $x$ is the scaled energy of the hadron. The model is then applied successfully to $\omega, \phi$ production in $p p$ collisions at the Relativistic Heavy Ion Collider, RHIC; these data form an important base-line for the study of Quark Gluon Plasma in heavy nucleus collisions at RHIC, and also in future at the LHC.
\end{abstract}

PACS numbers: 13.60.Le, 13.60.Hb, 13.66.Bc, 13.85.Ni 


\section{INTRODUCTION}

Studies of meson fragmentation are currently limited by data in comparison with the relatively abundant data available on deep inelastic processes (DIS). Meson fragmentation processes can be understood within QCD through time-like conjugates of the space-like processes that contribute to DIS. Hence there is a great deal of interest in the study of meson (and baryon) fragmentation. Data from $e^{+} e^{-}$collisions are most commonly available for pseudo-scalar and vector meson fragmentation. While e $p$ data is severely limited, there has recently been high quality data on pseudo-scalar meson production from $p p$ collision processes in RHIC[1]. Preliminary data on light mesons is already available from the LHC[2] and more is expected shortly. In particular, an understanding of $\eta$ and $\phi$ meson fragmentation in $p p$ processes is important as a baseline for the study of the production of these mesons in nucleus-nucleus collisions as a signal of quark-gluon plasma (QGP)[3]. While there exist many phenomenological studies on $\pi$ and $K \operatorname{meson}[4-10]$ and $\eta$ fragmentation[5, 11], as well as many comprehensive reviews $[6-8,17]$ of these, the issue of vector meson hadro-production has not been addressed so far. In this paper we focus attention for the first time on light $(u, d, s$ valence quarks only) vector meson fragmentation using a model that has earlier been applied to a study of light pseudo-scalar meson[4, 5] and octet baryon[4] fragmentation. In particular, the $\phi$ meson, which is almost a pure strange quark-anti-quark bound state, has special relevance as a signal for QGP[12-14].

A study of fragmentation functions requires an experimental input at a given $Q^{2}$ (momentum transfer) scale since QCD cannot predict the fragmentation functions themselves but only their $Q^{2}$ dependences. In the case of mesons formed from the light quarks $(u, d$, $s$ ), it is possible to apply symmetry arguments to reduce further the number of (unknown) input starting fragmentation functions.

A simple SU(3) symmetric model is introduced[4] which has been applied to pseudo-scalar octet mesons and octet baryons at the leading order level[4, 5]. In this paper, the above model is applied to study the fragmentation functions of octet vector mesons $\left(\rho\right.$ and $\left.K^{*}\right)$ in $e^{+} e^{-}$annihilation. The model reduces the various required input quark fragmentation functions to a combination of just three independent fragmentation functions, $\alpha\left(x, Q^{2}\right)$, $\beta\left(x, Q^{2}\right), \gamma\left(x, Q^{2}\right)$ and an $\mathrm{SU}(3)$ breaking scale-independent parameter $\lambda$ at a low input scale. In addition to these functions, the gluon and heavy quark contributions are also taken 
into consideration during evolution. The model is then extended to predict the octet-singlet mixing of $\omega, \phi$ mesons with a very simple ansatz that relates the single fragmentation function in the singlet sector to an octet fragmentation function along with a few additional constants. In contrast, other studies[6-11] in, say, the pseudo-scalar meson sector, fit individual data on different mesons with no attempt made to combine the data in any way whatsoever. This is in fact one possible reason why the vector meson sparse data set may have not been studied so far.

The paper is organized as follows: the kinematics of the relevant $e^{+} e^{-}$and $p p$ scattering processes is briefly explained in section 2. The model to study the fragmentation functions of vector mesons is introduced in section 3. The model is used to study the cross-section for hadro-production in section 4. In particular, a detailed study of the pure octet mesons, $\rho$ and $K^{*}$, is given in this section. It is then extended to include the singlet case, with singlet-octet mixing, in section 5. The detailed parameterization is explained in section 6 . Finally, the model is compared with both $e^{+} e^{-}$and $p p$ data in section 7 . A summary and discussion is presented in section 8 of the paper.

\section{FORMALISM}

\section{A. Hadron production in $e^{+} e^{-}$process}

The $e^{+} e^{-}$annihilation process is used to analyze the fragmentation functions of quarks (anti-quarks) through the hadrons which they produce. The reaction $e^{+} e^{-} \rightarrow h+X$ proceeds by first creating a quark and an anti-quark pair through $e^{+} e^{-} \rightarrow q \bar{q}$ via an intermediate vector boson $V=\gamma / Z^{0}$, followed by the fragmentation of the quark $(q)$, anti-quark $(\bar{q})$, or gluon $(g)$ into a hadron. This process is known as the fragmentation process[15].

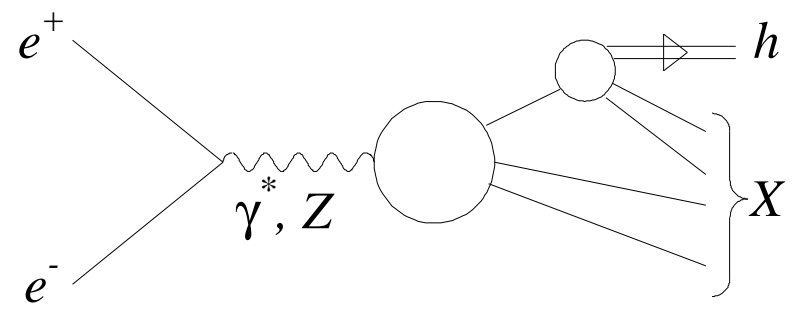

FIG. 1: A schematic diagram of the fragmentation process in $e^{+} e^{-}$scattering[16]. 
The term $h$ in Fig. 1 represents the hadron (vector meson in our case), where $X$ is the debris. The corresponding cross-section for this scattering process at c.m. energy $\sqrt{s}$ factorises into a component describing the hard scattering and one describing the hadronisation. Hence, it can be expressed to LO as[17]:

$$
\frac{1}{\sigma_{t o t}} \frac{\mathrm{d} \sigma^{h}}{\mathrm{~d} x}=\frac{\sum_{q} c_{q} D_{q}^{h}\left(x, Q^{2}\right)}{\sum_{q} c_{q}},
$$

in which the fragmentation function $D_{q}^{h}\left(x, Q^{2}\right)$ is the probability for a quark to hadronise to a hadron carrying a fraction $x$ of the energy from its parent quark, where $x \equiv E_{\text {hadron }} / E_{\text {quark }}=$ $\left(2 E_{h} / \sqrt{s}\right) \leq 1$ (or $\left.x_{p} \equiv 2 p_{h} / \sqrt{s}\right)$ and $\mathrm{Q}=\sqrt{s}$ is the energy scale, where we are considering the reaction.

The charge factors $c_{q}$ are associated with the quark $q_{i}$ with flavor $i$, written[17] in terms of the electromagnetic charge $e_{i}$, vector and axial vector electroweak couplings, $v_{i}=T_{3 i}-$ $2 e_{i} \sin ^{2} \theta_{\mathrm{w}}$ and $a_{i}=T_{3 i}$, as

$$
\begin{aligned}
c_{q} & =c_{q}^{V}+c_{q}^{A} \\
c_{q}^{V} & =\frac{4 \pi \alpha^{2}}{s}\left[e_{q}^{2}+2 e_{q} v_{e} v_{q} \rho_{1}(s)+\left(v_{e}^{2}+a_{e}^{2}\right) v_{q}^{2} \rho_{2}(s)\right], \\
c_{q}^{A} & =\frac{4 \pi \alpha^{2}}{s}\left(v_{e}^{2}+a_{e}^{2}\right) a_{q}^{2} \rho_{2}(s) \\
\rho_{1}(s) & =\frac{1}{4 \sin ^{2} \theta_{\mathrm{w}} \cos ^{2} \theta_{\mathrm{w}}} \frac{s\left(m_{Z}^{2}-s\right)}{\left(m_{Z}^{2}-s\right)^{2}+m_{Z}^{2} \Gamma_{Z}^{2}} \\
\rho_{2}(s) & =\frac{1}{\left(4 \sin ^{2} \theta_{\mathrm{w}} \cos ^{2} \theta_{\mathrm{w}}\right)^{2}} \frac{s^{2}}{\left(m_{Z}^{2}-s\right)^{2}+m_{Z}^{2} \Gamma_{Z}^{2}}
\end{aligned}
$$

The values of $T_{3 i}$, the third component of weak isospin, and $v_{i}$ are tabulated with weak mixing angle $\theta_{\mathrm{w}}$ in[18]. $\Gamma_{Z}$ and $m_{\mathrm{Z}}$ are the decay width and mass of the $Z$-intermediate gauge boson for high energy scale. We re-express the LO cross section in terms of singlet and non-singlet fragmentation functions, as

$$
\frac{1}{\sigma_{t o t}} \frac{\mathrm{d} \sigma^{h}}{\mathrm{~d} x}=\frac{\sum_{j} a_{j} D_{j}(x, t)}{\sum_{q} c_{q}},
$$

where $j=0,3,8,15,24$ and $D_{0}, D_{3}, D_{8}, D_{15}$ and $D_{24}$ refer to the singlet $\left(D_{0}=\sum_{i}\left(D_{i}+D_{\bar{i}}\right)\right)$, and the non-singlet $\left(n^{2}-1=3,8,15,24:(u-d),(u+d-2 s),(u+d+s-3 c)\right.$ and $(u+d+s+c-4 b) ; u \equiv D_{u}+D_{\bar{u}}$, etc.) combinations respectively. Here the coefficients are $a_{0}=\left(c_{u}+c_{d}+c_{s}+c_{c}+c_{b}\right) / 5, a_{3}=\left(c_{u}-c_{d}\right) / 2, a_{8}=\left(c_{u}+c_{d}-2 c_{s}\right) / 6, a_{15}=\left(c_{u}+c_{d}+c_{s}-3 c_{c}\right) / 12$ and $a_{24}=\left(c_{u}+c_{d}+c_{s}+c_{c}-4 c_{b}\right) / 20$. Note that the data from LEP or SLD on the $Z$ pole is dominated by the $D_{0}$ fragmentation function combination while at lower energies 
where photon exchange dominates, the $u$-type quark fragmentation functions dominate. Furthermore, data is available[26-29] only for the sum of the conjugate states, $\rho^{ \pm}=\rho^{+}+\rho^{-}$, and similarly for the charged and neutral $K^{*}$ mesons[30-33]. We therefore compare with the sums of these quantities. The SLD data[40, 41] is available separately for fragmentation from light quarks $(u, d, s)$ alone; hence for these data, the summation in Eq. (1) is over the light flavour contributions only. Data on the singlet-octet mixture of states, viz., $\omega$ and $\phi$, are available from both LEP and SLD[34-41].

\section{B. Hadron production in $p p$ process}

In addition to information on the unknown fragmentation functions, hadro-production in $p p$ processes requires a knowledge of the parton (both quark and gluon) distribution functions within the primary proton. The scattering is expressed in terms of the underlying parton interactions, with one of the final state partons fragmenting into the hadron of interest. All possible initial state parton interactions are possible: $q q, q g$ and $g g$, as well as processes with antiquarks.

The inclusive cross section for hadro-production, $p+p \rightarrow h+X$, producing a hadron $h$ at large $p_{T}$ is given by[19-21],

$$
E_{h} \frac{\mathrm{d}^{3} \sigma}{\mathrm{d} p_{h}^{3}}=\frac{1}{\pi} \sum \int_{x_{a}^{\min }}^{1} \mathrm{~d} x_{a} \int_{x_{b}^{\min }}^{1} \mathrm{~d} x_{b} P_{a}^{A}\left(x_{a}, Q^{2}\right) P_{b}^{B}\left(x_{b}, Q^{2}\right) \frac{\mathrm{d} \sigma^{a b \rightarrow c d}}{z_{h} \mathrm{~d} \hat{t}} D_{c}^{h}\left(z_{h}, Q^{2}\right)
$$

where the sum over $(a, b, c, d)$ runs over both quarks and gluons. Here $x_{a}$ and $x_{b}$ are the usual Bjorken- $x$ variables corresponding to the parent proton momenta $p_{A}$ and $p_{B}: x_{a}=$ $p_{a} / p_{A}, x_{b}=p_{b} / p_{B}$ (neglecting intrinsic transverse momentum) and $P_{a / A}\left(x_{a}, Q^{2}\right)$ are the usual parton density distributions; for example, $P_{u / p}\left(x_{a}, Q^{2}\right) \equiv u\left(x_{a}, Q^{2}\right)$, etc.

The fragmentation functions depend on the variables, $z=z_{h}=p_{h} / p_{c}$, the fraction of momentum of the quark carried by the fragmenting hadron, and the scale $Q^{2} \sim p_{T}^{2}$. The limits of integration are[19-21]

$$
x_{a}^{m i n}=\frac{x_{1}}{1-x_{2}} ; \quad x_{b}^{m i n}=\frac{x_{a} x_{2}}{x_{a}-x_{1}},
$$

with $x_{1}=-u / s, x_{2}=-t / s$.

For numerical comparison with the data, we reëxpress the cross-section in terms of the physical observables which are the transverse momentum $p_{T}=p_{h} \sin \theta$ and the rapidity 
$y=(1 / 2) \ln \left[\left(E_{h}+p_{h} \cos \theta\right) /\left(E_{h}-p_{h} \cos \theta\right)\right]$, as

$$
E_{h} \frac{\mathrm{d}^{3} \sigma}{\mathrm{d} p_{h}^{3}} \equiv \frac{1}{p_{T}} \frac{\mathrm{d}^{3} \sigma}{\mathrm{d} p_{T} \mathrm{~d} y \mathrm{~d} \phi},
$$

where $\theta$ is the scattering angle of the hadron $h$ in the $p p$ center of mass frame and $E_{h}$ and $p_{h}$ are its energy and 3-momentum. Note that the azimuthal angle dependence is trivial in this process; furthermore the data from the PHENIX experiment at RHIC is taken over a range $\pi$ in $\phi$ and $-0.35 \leq y \leq 0.35$ in the rapidity[12-14]. The sub-process cross-sections are well-known[19-22]; the $q q, q g$ and $g g$ processes all contribute at the same order in $\alpha_{s}$. Hence the quark and gluon fragmentation functions contribute at the same order, unlike in the $e^{+} e^{-}$case. This data is therefore an important test of the correctness of the gluon fragmentation functions.

We now present details of our model for quark fragmentation functions.

\section{SU(3) MODEL}

Fragmentation functions parameterize the hadronisation process, in which the observed hadrons are formed from the final state partons of the scattering process; these cannot be calculated in QCD. However, given a definite energy scale and starting distribution, QCD can evolve these perturbatively and explain their scale $\left(Q^{2}\right)$ dependence. A set of common fragmentation functions are used to describe the members of the octet of vector mesons $\rho\left(\rho^{+}, \rho^{-}, \rho^{0}\right), K^{*}\left(K^{*+}, K^{*-}, K^{* 0}, \bar{K}^{* 0}\right)$ and $\omega$. An $\mathrm{SU}(3)$ symmetric model (with parameterized $\mathrm{SU}(3)$ breaking) has been chosen to achieve this because, in principle, such a symmetry gives good description about the octet of vector mesons. Such a model with broken SU(3) was developed for pseudo-scalar octet $\pi$ and $K$ meson fragmentation in $e^{+} e^{-}$ collisions[5] and for the octet baryons [4] $p, n, \Lambda, \Sigma$. We use an analogous model in the vector meson sector in our analysis.

We start with light quarks $(u, d$ and $s)$ at the starting scale of $Q_{0}^{2}=1.5 \mathrm{GeV}^{2}$. However, as the fragmentation function evolves across various thresholds (typically up to $Q^{2}=(91.2$ $\mathrm{GeV})^{2}$ ), consistent contribution of charm and bottom quarks are included appropriately in the evolution. Let us consider the process at the input scale as,

$$
q_{i} \rightarrow h_{j}^{i}+X_{j}
$$


The underlying $\mathrm{SU}(3)$ process can be thought of as $3 \rightarrow 8+X$. That is, a quark goes to an octet hadron $\left(h_{j}^{i}\right)$ with the remainder $X_{j}$ being a triplet (3), antisixplet $(\overline{6})$ or fifteenplet (15) with $i, j$ running over 1 to 8 . Let $\alpha\left(x, Q^{2}\right), \beta\left(x, Q^{2}\right)$ and $\gamma\left(x, Q^{2}\right)$ be the corresponding unknown SU(3) symmetric independent fragmentation functions for each of these possibilities[4], that is, for $\mathrm{X}$ to be $3(\overline{6}, 15)$ the probability of the quark to fragment into an octet meson is $\alpha(\beta, \gamma)$.

In a similar way, an anti-quark also produces an octet hadron with $X_{j}$ being an antitriplet $(\overline{3})$, sixplet (6) or anti-fifteenplet $(\overline{15})$, for which $\bar{\alpha}\left(x, Q^{2}\right), \bar{\beta}\left(x, Q^{2}\right)$ and $\bar{\gamma}\left(x, Q^{2}\right)$ have to be determined.Thus a single meson has seven unknown fragmentation functions $D_{q}^{h}\left(x, Q^{2}\right), \bar{D}_{\bar{q}}^{h}\left(x, Q^{2}\right)$ and $D_{g}^{h}\left(x, Q^{2}\right)$ associated with its production. Here $D_{q}^{h}, \bar{D}_{\bar{q}}^{h}$ and $D_{g}^{h}$ refer to the light quark, anti-quark and gluon fragmentation functions while the heavier quark contributions are zero at the starting scale (below the charm threshold).

So, we have to fit a total of $56(8 \times 7)$ unknown fragmentation functions to the data for octet mesons, which is rather daunting. The problem is made simpler when we apply $\mathrm{SU}(3)$ symmetry since the seven independent symmetric fragmentation functions $\alpha\left(x, Q^{2}\right), \beta\left(x, Q^{2}\right)$ and $\gamma\left(x, Q^{2}\right)$ including their conjugates $\bar{\alpha}\left(x, Q^{2}\right), \bar{\beta}\left(x, Q^{2}\right)$ and $\bar{\gamma}\left(x, Q^{2}\right)$ and $D_{g}\left(x, Q^{2}\right)$, the gluon contribution, should determine the fragmentation of the entire group of octet mesons. Since SU(3) symmetry is only approximate, we use a single $x$-independent parameter to signify $\mathrm{SU}(3)$ breaking, while $\mathrm{SU}(2)$ remains unbroken in our model. Hence the fragmentation functions of $\rho^{ \pm}$and $\rho^{0}$ (and similarly for isospin conjugates of $K^{*}$ ) are related by isospin symmetry.

\section{A. Valence and Sea functions}

The vector meson octet is a self conjugate octet. So, $D_{q}^{h}=\bar{D}_{\bar{q}}^{h}$. Therefore, we have three independent fragmentation functions as mentioned in the above section and we express the quark fragmentation in terms of these three fragmentation functions in Table I. We reduce the number of unknown functions further through various symmetry considerations like isospin invariance and charge conjugation. We assume the sea is flavour symmetric, so that $D_{u}^{\rho-}=D_{s}^{\rho-}$ and so on. Using this assumption and the expressions for corresponding fragmentation functions given in Table I, we have

$$
\beta\left(x, Q^{2}\right)=\gamma\left(x, Q^{2}\right) / 2
$$


and all the sea fragmentation functions are equal to

$$
S\left(x, Q^{2}\right)=2 \gamma\left(x, Q^{2}\right)
$$

Thus, all valence fragmentation functions can be expressed in terms of the function $V\left(x, Q^{2}\right)$, where $V$ is given, say for $\rho^{+}$, by the difference $D_{u}^{\rho+}-D_{\bar{u}}^{\rho+}$. Therefore, we have

$$
V\left(x, Q^{2}\right)=\left(\alpha+\beta+\frac{3}{4} \gamma\right)\left(x, Q^{2}\right)-2 \gamma\left(x, Q^{2}\right)
$$

Substituting the value of $\beta$ from Eq. (7) in Eq. (9) we get the valence combination to be

$$
V\left(x, Q^{2}\right)=\alpha\left(x, Q^{2}\right)-\frac{3}{4} \gamma\left(x, Q^{2}\right)
$$

All sea fragmentation functions can be expressed in terms of $\gamma$. Thus, Eqs. (8) and (10) represent just two unknown fragmentation functions for the sea $S$ (or equivalently $\gamma$ ) and valence $V$, in terms of which all quark fragmentation functions which describe all the octet vector meson production can be represented.

\section{B. Breaking of SU(3) symmetry}

$\mathrm{SU}(3)$ symmetry is broken in the model due to relatively more massive strange quarks. So, in addition to valence and sea functions, we introduce an $x$-independent symmetry breaking parameter $\lambda$ for a non-strange quark to fragment into a strange octet meson.

For example, to produce $K^{*+}(u \bar{s})$ meson, a $u$ quark in the valence has to pick up a(n anti) $s$ quark; being more massive, the corresponding fragmentation function is suppressed by the parameter $\lambda$. Moreover, if the valence quark is $\bar{s}$ then it may easily pick up a $u$ quark without this suppression factor. In the same way, fragmentation of other mesons like $K^{*-}$, $K^{* 0}$ and $\bar{K}^{* 0}$ can be explained.

For all these mesons with strange quark in their valence, apart from different valence parts, their sea function remains the same $-2 \lambda \gamma$-because the sea is flavour symmetric and is uniformly suppressed by the suppression factor $\lambda$. Thus we use broken SU(3) symmetry with an SU(3) symmetric sea as our model to describe the fragmentation functions of vector mesons with the introduction of the parameter $\lambda$. 


\section{FRAGMENTATION OF $\rho$ AND $K^{*}$ MESONS}

We begin by neglecting the problematical $\omega$ meson that is not a pure $\mathrm{SU}(3)$ octet meson; it will be considered along with the $\phi$ meson later.

\section{A. $\rho$ meson}

The scattering cross section is expressed in terms of singlet and non singlet combinations in Eq. (3). Let us begin with $\rho$, the lightest meson, and express the fragmentation function for $u$ quark from Table I. On substituting the values of sea and valence parts from Eqs. (8) and (10) we get,

$$
D_{u}^{\rho^{+}}=V+2 \gamma
$$

as $u$ quark is present both in the valence as well as in the sea part and is equal to the $\bar{d}$ contribution. The charge conjugation invariance clearly shows that the other quarks are not in the valence of the $\rho$ meson; hence their fragmentation for sea part has to be $2 \gamma$. The singlet contribution at the input scale for $\rho$ meson (where only the three light flavours contribute) is therefore the total quark contribution:

$$
D_{0}^{\rho^{+}}=D_{u+\bar{u}+d+\bar{d}+s+\bar{s}}^{\rho^{+}}=2 V+12 \gamma
$$

The non singlet contributions $D_{3}$ and $D_{8}$ can be obtained for $\rho$ meson with the same analogy. Here, the non singlet term $D_{3}\left(=D_{u}-D_{d}\right)$ turns out to be zero due to charge conjugation invariance and $D_{8}=2 \mathrm{~V}$.

\section{B. $K^{*}$ meson}

The procedure for the $K^{*}$ meson is exactly the same as above. The only difference is that it has a strangeness quantum number. Thus for the valence component of $K^{*-}$ meson $(s \bar{u})$, the non-strange $u$ quark contribution is suppressed by $\lambda$ as it has to pick up a strange quark to form the $K^{*-}$ meson. However, the $s$ contribution is itself not suppressed, since only an (anti-) $u$ quark is required here. Meanwhile, all the sea fragmentation functions come with

a uniform factor of $\lambda$ since for all quarks (flavour symmetric sea), a strange quark has to be produced. Therefore,

$$
D_{0}^{K^{*+}}=D_{u+\bar{u}+d+\bar{d}+s+\bar{s}}^{K^{*+}}=(1+\lambda) V+12 \lambda \gamma
$$


Notice that while $\lambda$ is $x$-independent, there is an inherent $x$-dependence of the strangeness suppression, being maximal at small- $x$ and least at large- $x$ due to the different suppression of the valence and the sea quarks.

Thus $\rho$ and $K^{*}$ mesons have different valence and sea quark fragmentation functions. In addition, since the gluon fragmentation function mixes with the singlet $D_{0}$ fragmentation function on evolution, we parameterize a possible gluon suppression through $D_{g}^{K^{*}}=f_{g}^{K^{*}} D_{g}^{\rho}$.

\section{EXTENSION TO $\omega$ AND $\phi$ MESONS}

We now extend this broken $S U(3)$ model which explains in a most simple way the pure octet $\left(\rho\right.$ and $\left.K^{*}\right)$ mesons, to the $\omega$ and $\phi$ mesons, which are orthogonal combinations of the $\mathrm{SU}(3)$ octet $\left(\omega_{8}\right)$ and singlet states $\left(\omega_{1}\right)$ :

$$
\begin{aligned}
& \omega=\sin \theta \omega_{8}+\cos \theta \omega_{1}, \\
& \phi=\cos \theta \omega_{8}-\sin \theta \omega_{1} .
\end{aligned}
$$

where $\omega_{8}=(u \bar{u}+d \bar{d}-2 s \bar{s}) / \sqrt{6}, \omega_{1}=(u \bar{u}+d \bar{d}+s \bar{s}) / \sqrt{3}$ are the corresponding orthogonal states and $\theta$ is the vector mixing angle, whose value is approximately[23] $35^{\circ}$. Note that a value of $\theta$ close to this value saturates the physical $\phi$ state[24] as a pure $s \bar{s}$ state. The fragmentation functions for $\omega_{8}$ can be described with the help of fragmentation functions given in Table I, since $\omega_{8}$ is one of the members of the octet, whereas details regarding $\omega_{1}$ will be discussed in the next section.

\section{A. Singlet hadron $\left(\omega_{1}\right)$ fragmentation}

Let us consider the same process,

$$
q_{i} \rightarrow h+X_{i}
$$

in which a quark hadronises into a singlet meson so that $X$ can only be a triplet $(3 \rightarrow 1+X)$. Therefore, we need to determine only one unknown fragmentation function $\delta\left(x, Q^{2}\right)$ in the singlet case. In section 3 we saw that the probability for a parton to fragment into an octet hadron with $X$ being triplet is $\alpha\left(x, Q^{2}\right)$. For fragmenting to a meson so that the remainder $X$ is a triplet state, therefore, there are only two possibilities: either the hadron is an octet 
(the process is proportional to $\alpha$ ) or the hadron is a singlet (the process is proportional to $\delta$ ). Hence we use the simple ansatz that the function $\delta$ is simply related to $\alpha\left(x, Q^{2}\right)$, the fragmentation function for members of octet meson. That is,

$$
\frac{\delta}{3}=\frac{f_{1} \alpha}{3}=\frac{f_{1}}{3}\left(V+\frac{3}{4} \gamma\right),
$$

where the factor of $1 / 3$ is due to the normalization of the state and $f_{1}$ is the (presumed) $x$-independent proportionality constant we have to determine in the analysis. Since this uses an approximate $\mathrm{SU}(3)$ symmetry, this constraint is applied only at the input scale $Q_{0}^{2}$ where there are only three active flavours. The evolution is correctly applied to all the active flavours, depending on the scale.

We will now express the fragmentation of $\omega$ and $\phi$ mesons in terms of the SU(3) octet and singlet contributions.

\section{B. Singlet and octet contribution to $\omega$ and $\phi$ mesons}

The strangeness suppression factor $\lambda$ remains the same here. However, there is a difference in the sea suppression factor. While it was also equal to $\lambda$ in the case of the $K^{*}$ meson, here the suppression is rather different. It arises due to the preference of a given $q \bar{q}$ pair to fragment to the lighter $\rho$ rather than to $\omega$ or $\phi$. In particular, in the case of $\omega$, which is mostly saturated by non-strange quarks in the valence, the sea suppression factor arises due to the preference of a given light $q \bar{q}$ pair to fragment to the lighter $\rho$ rather than the $\omega$. Hence the sea suppression factor is expected to be $f_{\text {sea }}^{\omega} \sim m_{\rho}^{2} / m_{\omega}^{2}$ just as in the case of pseudoscalar mesons[5], and we do not expect a large suppression. On the other hand, the physical $\phi$ state is saturated by the strange contribution; hence the suppression factor $f_{\mathrm{sea}}^{\phi}$ for $\phi$ is expected to be close to $\lambda^{2}$ as a quark has to pick up both $\mathrm{s}$ and $\bar{s}$ from flavour symmetric sea. We will see in the next section that numerical fits to the data do indeed agree with these expectations.

We first explicitly write down the fragmentation functions given in Table I for corresponding octet hadrons in terms of valence and sea sectors, including the various suppression factors as discussed above:

$$
\begin{aligned}
D_{u}^{8} & =\frac{V}{6}+2 f_{\text {sea }} \gamma, \\
D_{s}^{8} & =\frac{2}{3} \lambda V+2 f_{\text {sea }} \gamma ;
\end{aligned}
$$


where the sea $\gamma$ and valence $V$ have their usual definitions, described in Eqs. (8) and (10), $\lambda$ is the strangeness suppression factor while $f_{\text {sea }}$ is the unknown suppression factor for the $\mathrm{SU}(3)$-symmetric sea fragmentation functions.

Using our ansatz for the singlet hadron, we have

$$
\begin{aligned}
D_{u}^{1}=D_{d}^{1} & =\frac{f_{1}^{u}}{3}\left(V+\frac{3}{4} f_{\mathrm{sea}} \gamma\right), \\
D_{s}^{1} & =\frac{f_{1}^{s}}{3}\left(\lambda V+\frac{3}{4} f_{\mathrm{sea}} \gamma\right) .
\end{aligned}
$$

Here we have introduced separate suppression factors for the $u, d$ - and $s$-type singlet fragmentation functions. With these four equations in hand, we express the fragmentation functions for $\omega$ and $\phi$ mesons, given the definitions of the states in Eq. (13), with vector mixing angle $\theta$, at the input scale as,

$$
\begin{aligned}
& D_{i}^{\phi}=\left(c_{i}^{\phi}\right)^{2}\left(\cos ^{2} \theta \frac{D_{i}^{8}}{\left(c_{i}^{8}\right)^{2}}+\sin ^{2} \theta \frac{D_{i}^{1}}{\left(c_{i}^{1}\right)^{2}}\right) ; \\
& D_{i}^{\omega}=\left(c_{i}^{\omega}\right)^{2}\left(\sin ^{2} \theta \frac{D_{i}^{8}}{\left(c_{i}^{8}\right)^{2}}+\cos ^{2} \theta \frac{D_{i}^{1}}{\left(c_{i}^{1}\right)^{2}}\right) .
\end{aligned}
$$

Here, $i$ refers to the three light quarks $(u, d, s)$; the co-efficients are $c_{u}^{\phi}=c_{d}^{\phi}=(\cos \theta-$ $\sqrt{2} \sin \theta), c_{s}^{\phi}=(-2 \cos \theta-\sqrt{2} \sin \theta)$ and $c_{u}^{8}=1, c_{s}^{8}=2, c_{u}^{1}=c_{s}^{1}=\sqrt{2}$. Obviously, we can find the coefficients for $\omega$ meson in the same way[5]. These fragmentation functions can be re-expressed in terms of $D_{0}^{\omega}\left(x, Q^{2}\right)$, etc., in the usual way. Finally, we again parameterize the gluon fragmentation functions as $D_{g}^{\omega, \phi}=f_{g}^{\omega, \phi} D_{g}^{\rho}$.

\section{PARAMETERIZATION OF THE INPUT FRAGMENTATION FUNCTIONS}

The unknown functions for the valence $V\left(x, Q^{2}\right)$, sea $\gamma\left(x, Q^{2}\right)$, and gluon $D_{g}\left(x, Q^{2}\right)$ fragmentation are parameterized at low input scale of $Q_{0}^{2}=1.5 \mathrm{GeV}^{2}$ for three light quarks $(u$, $d, s)$ where the charm and bottom contributions $\left(D_{c}, D_{b}\right)$ are zero. The parameters are then determined through fits with data. We use a standard functional form to describe these quantities:

$$
F_{i}(x)=a_{i} x^{b_{i}}(1-x)^{c_{i}}\left(1+d_{i} x+e_{i} x^{2}\right),
$$

where $a_{i}, b_{i}, c_{i}, d_{i}$ and $e_{i}$ are the values to be determined. The fragmentation functions are evolved to leading order (LO) including charm and bottom contributions in appropriate places along with the gluon fragmentation, to the $Q^{2}$ values of interest. Since the gluon 
fragmentation does not occur in the expression for the cross-section, it is least constrained by these fits and relatively unknown. Hence we set $e_{g}=0$ for the gluon.

\section{DATA ANALYSIS AND RESULTS}

\section{A. Fragmentation in $e^{+} e^{-}$process}

We therefore have a set of parameterizations as well as a bunch of scale-independent constants that need to be determined. We focus here entirely on the $e^{+} e^{-}$data as the cleanest sample. Hence necessarily the fits to the gluons are ill-determined, since, to LO, the gluon contributes only through evolution. Furthermore, we concentrate on the $Z$-pole data[25] from both LEP as well as SLAC-SLD. At this scale, five quark $(q \bar{q})$ flavour pairs from $u$ to $b$ are produced in the final state and hence contributions to the final state meson are of two types: first, is the direct fragmentation of the quark (anti-quark) into a vector meson; another is the fragmentation of a heavy (for example $D$ or $B$ ) meson which subsequently decays into one of the mesons of interest. Since $b$ quarks decay dominantly (greater than 95\% of the time) into $c$-quarks, the decay chain of both heavy $b$ and $c$ end up in strange mesons, driven by the large $V_{c s}$ CKM matrix element. We therefore expect that data on the strangeness containing $K^{*}$ and $\phi$ mesons will have large contamination from these heavy flavour decays.

On the other hand, the $K$ mesons decay predominantly into $\pi$ and $\eta$; hence there is very little contamination of the $\rho$ and $\omega$ data. In this analysis, therefore, we consider the inclusive hadro-production data from LEP on the predominantly non-strange vector mesons, $\rho$ and $\omega[24]$ to arise from light quark fragmentation. For the strange mesons, $K^{*}$ and $\phi$, we use instead the so-called $u d s$ data from SLD where the fragmentation of the light quarks alone have been segregated and studied.

\section{For pure octet mesons, $\rho$ and $K^{*}$}

For extraction of fragmentation functions from the data we used inclusive particle production in $e^{+} e^{-}$as the process of choice. The unknown fragmentation functions $V\left(x, Q^{2}\right)$, $\gamma\left(x, Q^{2}\right), D_{g}\left(x, Q^{2}\right)$ and the suppression factor $\lambda$ are determined by comparison with the $\operatorname{data}[25]$. 
The comparison with data is restricted to the range $x \gtrsim 0.01$ since it is well-known that the DGLAP evolution does not explain the behaviour of the cross-section at small- $x$ and modifications such as MLLA (modified leading log approximation) are needed to explain the small- $x$ data.

Note that individual data sets are some-what small, with typically $6-8 x$-bins. This is insufficient to fit all the unknown fragmentation functions (quark and gluon). However, the model can be applied to the entire octet meson data as a whole; this allows for a reasonable fit to be obtained with available data. This, in fact, is the primary motivation for constructing such a model.

Using the available data we fit the functional form of Eq. (18) for pure octet mesons ( $\rho$ and $K^{*}$ ) by fixing the values of parameters $a, b, c, d$ and $e$ for valence, sea and gluon fragmentation functions.

The smaller $x$ behaviour is dominated by the sea $(\gamma)$ contribution and large $x$ behaviour by the valence $V$ contribution; this helps determine the values of $a, b, c$. This process is not very sensitive to the gluon contribution since this contributes only in the evolution and not directly in the definition of the cross-section at LO, unlike in, say, the $p p$ process.

a. The fragmentation functions : The best-fit values of the parameters in the input fragmentation functions and the $1-\sigma$ errors on them are tabulated in Table II. These correspond to the input fragmentation functions for $\rho$ mesons having three light quarks (the heavier $c, b$ quarks do not contribute) at the starting scale of $Q_{0}^{2}=1.5 \mathrm{GeV}^{2}$, as shown in Fig. 2. This figure clearly shows that the valence contribution at large $x$ is dominated by $u$ and $d$ flavours, while strange flavour and gluon contribute only in the small $x$ sea part. As the evolution crosses various thresholds the charm and bottom contributions are included appropriately as depicted in Fig. 2 which clearly reflects the (small) charm and bottom contribution after evolution at the $Z$-pole. The heavy flavour contribution purely arises from gluon-initiated processes and cannot account for the contribution in inclusive hadro-production data arising from production and decay of heavy flavour mesons.

b. The $\rho, K^{*}$ cross-sections at the $Z$-pole : Given these fragmentation functions, finally we plot the various cross-section combinations as given in Eq. (1) along with data[26-28] for the $\rho$ meson in Fig. 3. The $\chi^{2}$ values of the fits (obtained by averaging the cross-section over each bin and comparing with the data) are tabulated in Table IV. (The difference between the average cross-section and its value at the average $x$ value of the bin indicated 
the uncertainty due to bin width and has been included as an error in the computation. This affects the $\chi^{2}$ significantly only in the last bin).

We also use the fitted values to predict and compare with data on $\rho$ meson fragmentation at the photon-exchange-dominated regime of $\sqrt{s}=29 \mathrm{GeV}$; see Fig. 3. Note that the $\gamma$ exchange process at lower energies is sensitive to a different combination of the fragmentation functions than at the $Z$-pole.

The value of the suppression factor can be determined by the fraction of $\rho$ and $K^{*}$ meson at small $x$ (the data at large $x$ have relatively larger error bars). A fit to the data as seen in Fig. 4 gives $\lambda=0.063$ (see Table III). Notice that the $1-\sigma$ range of $\lambda(0.05 \leq \lambda \leq 0.07)$ for the vector meson octet is close to that obtained $(\lambda \sim 0.08)$ for strangeness suppression of the pseudo scalar mesons $K^{ \pm}, K^{0}$ and $\overline{K^{0}}$. This may indicate that the origin of strangeness suppression may be independent of the spin structure of the mesons, since the two octets are otherwise unrelated.

The data[40, 41] for $K^{*}$ meson is also plotted along with our predictions, using the same input parameters as used for the $\rho$ meson, in Fig. 4 with the best fit value of the suppression factor for gluon, $f_{g}^{K^{*}}=1.0$ (error bar is in Table III). The data are reasonably well fitted over a large $x$ region. Thus all the parameters in the input fragmentation functions are completely determined from fits to the $\rho$ and $K^{*}$ meson data. The $\rho$ data gives a set of $V$, $\gamma\left(\right.$ and $D_{g}$ ), which consistently fit the $K^{*}$ data with the inclusion of the two parameters $\lambda$
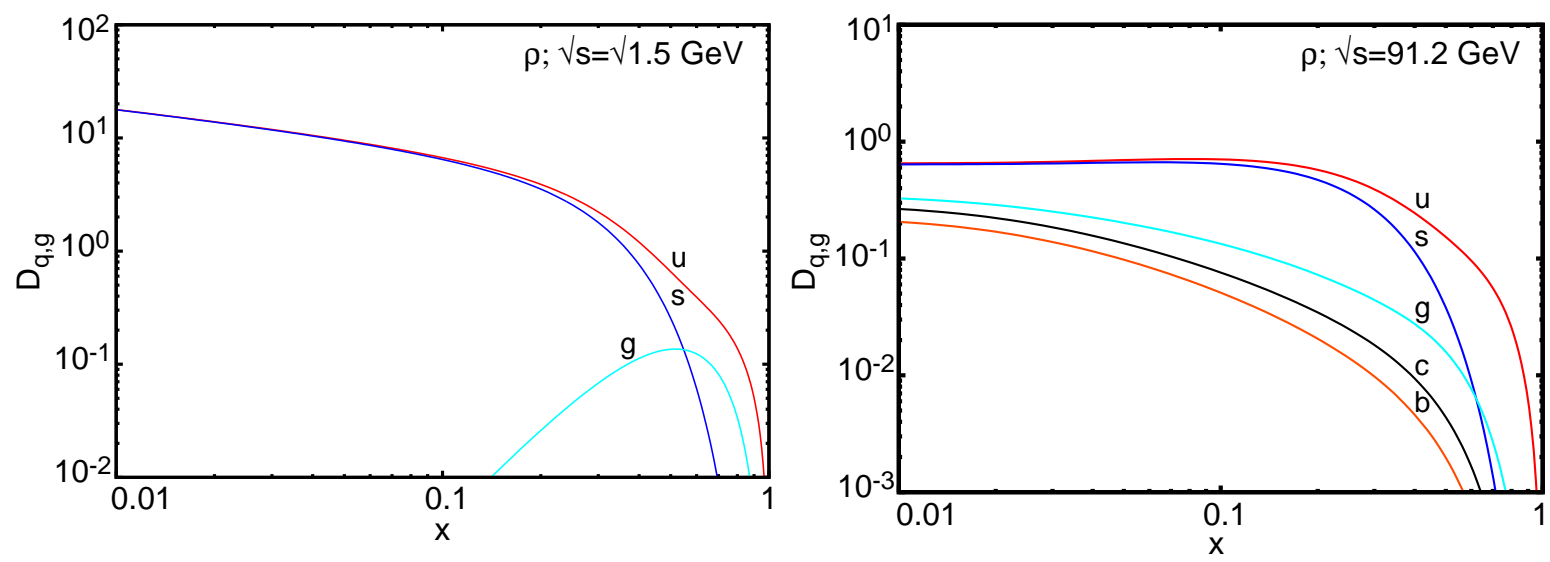

FIG. 2: (L) Three input flavours $u=d$ and $s$ with gluon $g$ at the starting scale of $\sqrt{s}=\sqrt{1.5} G e V$, as a function of $x$, with zero contribution of $c$ and $b$ flavours for $\rho$ meson. (R) Contribution of all the five flavours $u=d, s, c$ and $b$ with $g$ after leading order evolution at a scale of $\sqrt{s}=91.2 \mathrm{GeV}$ for $\rho$ meson. 
and $f_{g}^{K^{*}}$. Note that $\lambda$ contributes differently at low and high $x$ : the suppression factors are $(1+\lambda) / 2$ and $\lambda$ for the valence and sea quark fragmentation functions compared to those for $\rho$ fragmentation, as seen by a comparison of Eqs. (11) and (12). Hence the excellent fits to the $\rho$ and $K^{*}$ meson data validate our simple model in a non-trivial way.

Thus using the available data for $\rho$ and $K^{*}$ mesons we have fitted the input fragmentation functions as listed in Table II using a simple broken SU(3) model. We now go on to apply the model in the singlet-octet mixed $\omega-\phi$ sector, with the fragmentation functions $V, \gamma$, and $\lambda$ fixed at the values obtained from fitting the $\rho, K^{*}$ data.

\section{For mesons of octet and singlet mixture}

As our model explains pure octet mesons in a nice fashion, we extended it to mesons having mixture of octet and singlet components with same fragmentation functions $V, \gamma$, $D_{g}$ and breaking parameter $\lambda$. An additional fragmentation function expresses the singlet contribution. However, this is simply related to a known octet fragmentation function, as discussed earlier, and hence only a few additional constant parameters are introduced in this extended sector, namely $f_{1}^{u}, f_{1}^{s}, f_{\text {sea }}$ and $f_{g}$ for $\omega$ and $\phi$.

These describe the suppression in the sea as well as relate the singlet fragmentation function to the octet one. With the help of Eqs. (16), (17) and (18) the fragmentation
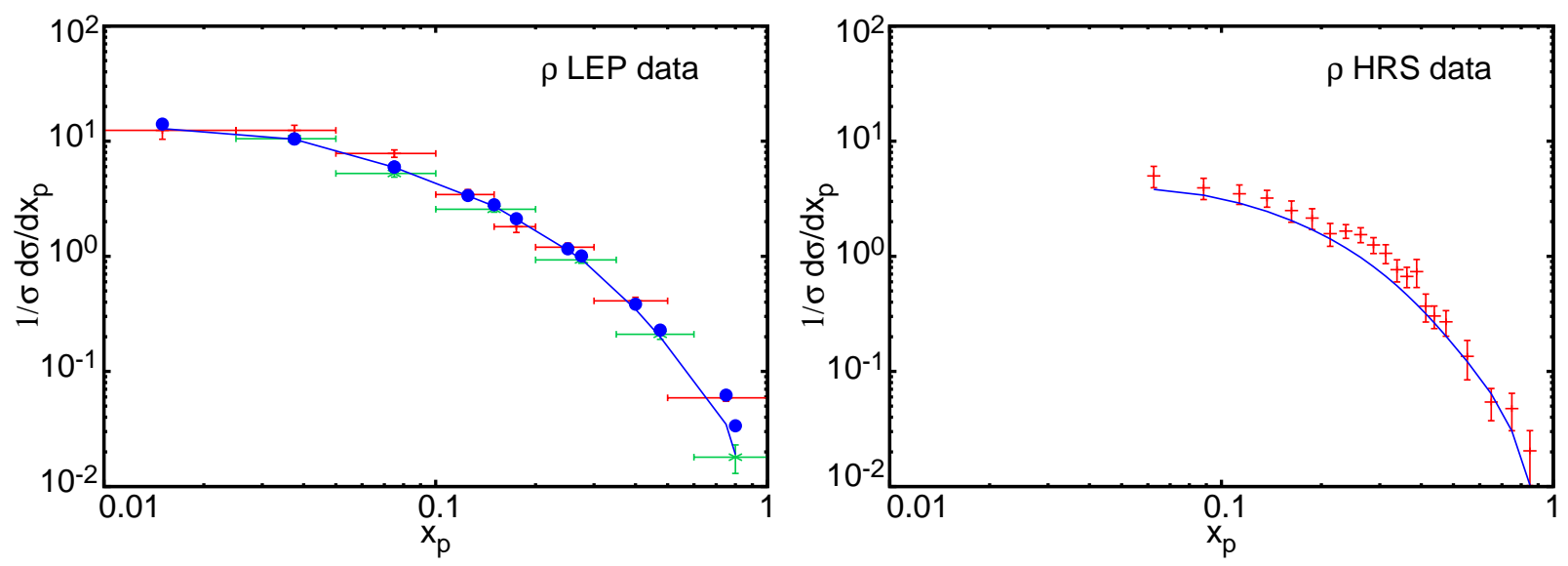

FIG. 3: Fit for rho meson in terms of fragmentation functions with (L) LEP data on the $Z$-pole and (R) HRS data at $\sqrt{s}=29 \mathrm{GeV}$. The data[26-28] at the $Z$-pole and from HRS[29] are shown with statistical and systematic errors added in quadrature while the dots in the left-side plot show the fit when averaged over the same $x$ bins as the data. 
functions for $\omega$ and $\phi$ mesons are expressed in terms of those of the $\omega_{8}$ and $\omega_{1}$ mesons, including these unknown parameters. The first is that of the mixing angle, $\theta$. It is known that $\theta$ is large and positive[24], close to $35^{\circ}$. Exactly at $35^{\circ}$, the $\omega$ meson is almost purely non-strange while the $\phi$ is almost purely a strange $s \bar{s}$ hadron. A simultaneous best fit to $\omega$ and $\phi$ data (keeping the $V, \gamma$ and $\lambda$ fixed to the best fit values from the $\rho$ and $K^{*}$ analysis) gives $\theta=42.6^{\circ}$ (error bar is in Table III), not far from maximal. Hence the strange (nonstrange) quark contribution to $\omega(\phi)$ is highly suppressed (the coefficients $\left(c_{s}^{\omega}\right)^{2}$ and $\left(c_{u}^{\phi}\right)^{2}$ are just a few percent of $\left(c_{u}^{\omega}\right)^{2}$ and $\left(c_{s}^{\phi}\right)^{2}$ respectively.) Note the data are inconsistent with no mixing, $\theta=0^{\circ}$.

Hence the $\omega$ meson is totally dominated by $u$ and $d$ light quarks. So it only has contribution from these two flavours whereas strangeness has least contribution. Therefore, we expect this to be similar to $\rho$, as is borne out by the similarity in cross-section behaviour. Since the strange component of $\omega$ is highly suppressed, we fix the strange singlet suppression factor to be $f_{1}^{s}=0$ for $\omega$. We find the data fit best to a sea suppression factor $f_{\text {sea }}^{\omega}=0.94$ and $f_{g}^{\omega}=1.0$ with $f_{1}^{u}$ consistent with zero (error bars are in Table III). This clearly shows that $\omega$ behaves like $\rho$, with unsuppressed sea and gluon fragmentation functions and very little contamination to the non-strange fragmentation functions from the singlet mixing, that is, the singlet contribution merely serves to make $\omega$ a practically non-strange meson orthogonal to $\rho$.

Similarly, we set $f_{1}^{u}=0$ for $\phi$. Since $\phi$ is mostly saturated by the strange contribution,

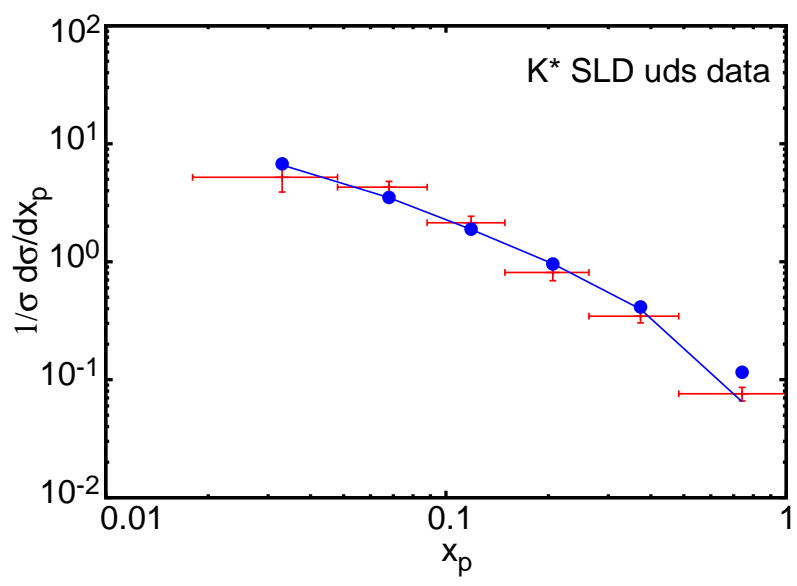

FIG. 4: Fit for $K^{*}$ meson with the best fit value of suppression factor $\lambda=0.063$. Data[40, 41] at the $Z$-pole, from light quarks only, are also shown. See caption of Fig. 3 for more details. 
the sea fragmentation function involves picking up both $s$ and $\bar{s}$ quarks and we therefore expect the sea suppression factor to be of the order of $\lambda^{2}$. Since the best-fit results were close to this value, with large error bars, we simply set $f_{\text {sea }}^{\phi}=\lambda^{2}$. Meanwhile, the best fit value of the singlet constant is $f_{1}^{s}=4.0$. The gluon suppression factor for $\phi$ is $f_{g}^{\phi}=0.32$ (error bars are in Table III). Hence, in contrast to the other member of the nonet, $\phi$ is heavily suppressed both with respect to sea quark and gluon fragmentation. These best-fit values result in a cross-section behaviour as shown in Fig. 5.

A detailed analysis of the gluon contribution and its relative suppression in $\phi$ mesons is best done in a next-to-leading order (NLO) analysis where the gluon fragmentation function directly appears in the expressions for the cross-section. An alternative is to study hadro-production in $p p$ scattering, where gluon fragmentation is equally dominant as quark fragmentation due to the type of parton-level processes involved, as stated earlier, although there are extra uncertainties due to convolutions with parton density distributions in the cross-section formulae. We therefore apply the fits obtained from hadro-production in $e^{+} e^{-}$ processes, with a clean (non-hadronic) initial state, to hadro-production in $p p$ collisions.

\section{B. Fragmentation in $p p$ process}

The PHENIX experiment at RHIC has measured $\omega$ and $\phi$ vector meson production[1214] in $p p$ collisions at $\sqrt{s}=200 \mathrm{GeV}$ as a function of the transverse momentum, $p_{T}$. We compute the relevant hadro-production cross-section as expressed in Eqs. (4) and (6). We
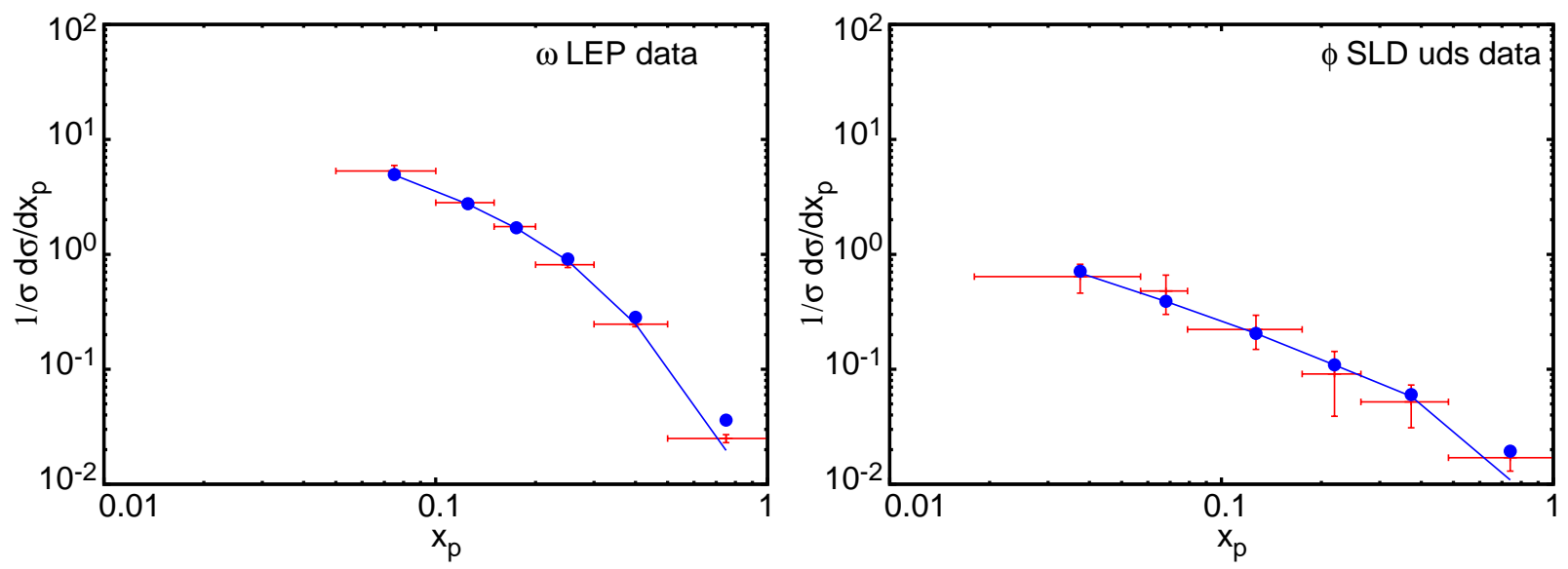

FIG. 5: Fits to omega (L) and (R) phi meson. The data correspond to LEP data[34, 35] for $\omega$ and the SLD data[40, 41] from light quarks alone for $\phi$. See the caption of Fig. 3 for more details. 
integrate over a range $\pi$ in the azimuthal angle $\phi$ and over a rapidity range $-0.35 \leq y \leq 0.35$ as stated earlier, and compare the cross-sections, computed at a scale $Q^{2}=p_{T}^{2}$, with the $p_{T^{-}}$ dependent data. We use (GRV98-LO)[42], a standard set of parton distributions as available in the CERN-libraries; a different choice of parton distributions will not affect the results since the $\left(x, Q^{2}\right)$ range of the data are in the well-studied range. The data are binned in $p_{T}$ and the cross-sections are quoted at the central value of the bin. As with $e^{+} e^{-}$data, the difference between the average cross-section and the cross-section value at the average $p_{T}$ of the bin is included in the error while computing the $\chi^{2}$ of the fits. Note that the fragmentation functions are taken from the fits to the $e^{+} e^{-}$data and there are no more free parameters.

The results of the computation are shown for both $\omega$ and $\phi$ mesons in Fig. 6 in comparison with data for which the scale is larger than the starting scale of evolution, $p_{T}^{2}>Q_{0}^{2}$. The figure also shows the band due to the scale uncertainty over the range $p_{T}^{2} / 2 \leq Q^{2} \leq 2 p_{T}^{2}$. It is seen that the model provides a good fit to the data. The values of $\chi^{2}$ are listed in Table IV corresponding to the central $Q^{2}$ value, $Q^{2}=p_{T}^{2}$.

\section{SUMMARY AND DISCUSSION}

Fragmentation functions of quarks and gluon for octet vector mesons are studied for the first time in inclusive hadro-production in electron-positron annihilation process and
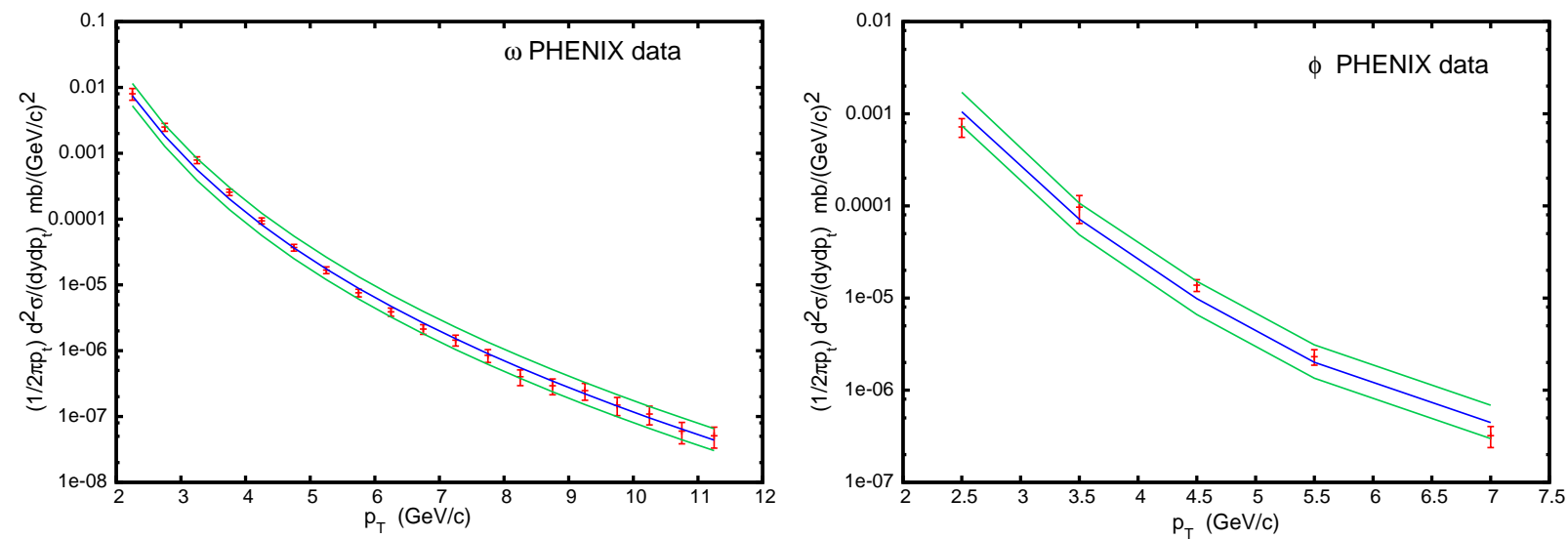

FIG. 6: Cross section as a function of $p_{T}$ for omega (L) and phi (R) meson hadro-production in $p p$ collisions. The data correspond to PHENIX/RHIC data[12-14] for $\omega$ and $\phi$. Bands show the scale uncertainty on changing $Q^{2}=p_{T}^{2}$ over a range $p_{T}^{2} / 2$ (upper curve) $\leq Q^{2} \leq 2 p_{T}^{2}$ (lower curve). 
proton-proton collisions to the leading order in QCD.

The unknown fragmentation functions were fitted to data on the $Z$ pole in $e^{+} e^{-}$collisions. The model fairly depicts the octet mesons $\rho, K^{*}$ and $\omega$ and greatly reduces the number of unknown fragmentation functions since the symmetries reduce the 48 quark fragmentation functions of the meson octet to combinations of just two: the valence $V\left(x, Q^{2}\right)$ and sea $\gamma\left(x, Q^{2}\right)$ fragmentation functions, apart from the gluon $D_{g}\left(x, Q^{2}\right)$ fragmentation function. This gives the model great predictability, since the various (sparse) data sets can be effectively combined to improve the quality of the fits.

An $\mathrm{SU}(3)$ breaking parameter $\lambda$ was introduced for the strange $K^{*}$ mesons phenomenologically, to account for strangeness suppression, at the starting scale of evolution. The parameters at the input scale were determined by comparison with data on the $Z$ pole. Heavy quark contributions that are radiatively generated, are small and seen to contribute at smaller $x$ values. The input distributions were tuned to give good fits with inclusive hadroproduction data at LEP for $\rho$ mesons and the "pure uds" (tagged jet) $K^{*}$-production data at SLD on the $Z$-pole. The best-fit values, along with the $1-\sigma$ errors are listed in Table II. The best fit value of the strangeness suppression factor is $0.05 \leq \lambda \leq 0.07$, close to the similar suppression factor obtained in fits to the pseudoscalar octet mesons, $\lambda \sim 0.08$, indicating that strangeness suppression in quark fragmentation functions may be a spin-independent phenomenon.

The model was extended with some further assumptions to include a study of the singlet vector meson with singlet-octet mixing (that is, to the $\omega$ and $\phi$ mesons). While the mixing angle is known to be close to $\theta \sim 35^{\circ}$, its value was determined through a simultaneous best fit to the $\omega$ and $\phi$ data to be $\theta \sim 43^{\circ}$ (see Table III). This is ultimately the focus of this work since $\eta$ and $\phi$ hadro-production in $p p$ collisions are used as a baseline when studying the production of these mesons as a signal of quark gluon plasma in nucleus-nucleus collisions.

In order to describe the singlet sector, an ansatz was made, relating the singlet fragmentation functions to one of the octet fragmentation functions $\left(\alpha\left(x, Q^{2}\right)\right)$. The constants of proportionality, $f_{1}^{q}$, and the gluon suppression factors were determined through the fits. While extending this model to include the octet and singlet nonet, no new fragmentation functions were introduced: just a few more parameters were included as described above, as well as the parameter $f_{\text {sea }}$ to describe suppression of sea quark fragmentation in both $\omega$ and (especially) $\phi$. 
The proportionality constants $f_{1}^{u}$ and $f_{1}^{s}$ and the sea suppression factor $f_{\text {sea }}$ were determined by fitting the functions with the data. Values of the parameters obtained appear to be phenomenologically reasonable and are listed in Table III, along with the 1- $\sigma$ errors, while the $\chi^{2}$ of the fits are given in Table IV.

Note that the input fragmentation functions at a low energy scale, $\left(Q_{0}^{2}=1.5 \mathrm{GeV}^{2}\right)$ were included for three light flavours $(u, d, s)$ only. The heavier flavours charm and bottom are consistently included at the appropriate thresholds during (leading order) evolution to the $Z$ pole. As is well known, next-to-leading order corrections will worsen the fits at small- $x$ unless mass corrections are added. This is beyond the scope of the present work.

Note also that the contribution at the $Z$-pole is dominated by the flavour singlet fragmentation function $D_{0}$, which is the sum of the individual quark flavour contributions with equal weight. Hence the fits are most sensitive to this sum rather than to the individual fragmentation functions. However, within the model, there are only two independent fragmentation functions, a valence and a sea combination, in terms of which all quark fragmentation functions are expressed. These appear with different weights in the cross-sections for $\rho$ and $K^{*}$ production and hence can be individually determined.

At LO, the fits are rather insensitive to gluon fragmentation, although it appears that the gluon fragmentation of $\phi$ is significantly suppressed related to the others. Also, we have not included any isospin breaking effects. In particular, charge asymmetries in fragmentation functions are best studied through fragmentation in $e p$ scattering, while $p p$ processes are sensitive to the gluon fragmentation. However, data, as well as analysis, is not as clean in this sector due to uncertainties in scale, for instance, in the $p p$ case. Hence fits to the fragmentation functions from data in $e^{+} e^{-}$sector can then be used as constraints while analyzing data from these other processes. Such an analysis was done for $\omega$ and $\phi$ hadroproduction in $p p$ collisions and was found to be in good agreement with RHIC/PHENIX data. Note that there are no free parameters in this fit since all the fragmentation functions are determined from earlier fits to the LEP data. Reasonable values of $\chi^{2}$ were obtained, as listed in Table IV, although the scale dependences are quite severe. This is expected to improve at next-to-leading order, although again this is beyond the scope of the present work.

In summary, fragmentation of the entire nonet of vector meson is explained via a simple model with broken $\mathrm{SU}(3)$. The model includes drastically few fragmentation functions and 
some constant parameters at a low input scale which were then evolved to the scale of the data (mainly at the $Z$-pole). While the fits to the pure octet vector mesons were very good, reasonable fits were obtained when the model was extended to study the mixed $\omega$ and $\phi$ mesons. The model continued to give good fits to the hadro-production data in $p p$ collisions as well, where the gluon fragmentation becomes important. This reflects the great predictability and efficiency of this model, especially in view of the paucity of data in this sector.

\section{Acknowledgements}

We thank M V N Murthy for discussions and feed-back. One of the authors HS would like to thank Professor A.S. Vytheeswaran, University of Madras, for his motivation and suggestions. The author is also thankful to the University of Madras for financial support in the form of a University Research Fellowship (URF).

[1] A. Adare et al., RHIC Collab., Phys.Rev. D 83032001 (2011).

[2] Sridhara Dasu, Rediscovering Standard Model at LHC, talk at Workshop on Synergy between High Energy and High Luminosity Frontiers, January 10-12, 2011, Tata Institute of Fundamental Research, Mumbai, India.

[3] J. D. Tapia Takaki, for the ALICE Collab., J. Phys. G 35, 044058 (2008).

[4] D. Indumathi, H. S. Mani, A. Rastogi, Phys. Rev. D 58, 094014 (1998); arXiv: hepph/9802324v1, 1998.

[5] D. Indumathi, B. Misra, arXiv:0901.0228v1, 2009.

[6] S. Albino, Rev. Mod. Phys. 82, 2489 (2010).

[7] S. Albino, E. Christova, Phys. Rev. D 81094031 (2010), arXiv:1003.1084.

[8] S. Albino, E. Christova, E. Leader, arXiv:1102.2305 (2011).

[9] M. Hirai, S. Kumano, Prog. Theor. Phys. Suppl. 186, 244 (2010); arXiv:1008.3814.

[10] M. Hirai, S. Kumano, T.-H. Nagai, K. Sudoh, Phys. Rev. D 75, 094009 (2007); arXiv:hep$\mathrm{ph} / 0702250$.

[11] C. A. Aidala, F. Ellinghaus, R. Sassot, J. P. Seele, M. Stratmann, Phys. Rev. D 83, 034002 
(2011); arXiv:1009.6145.

[12] A. Adare et al., RHIC Collab., Phys.Rev. D 83052004 (2011); see also details (especially of $p_{T}$ bins) in $[13,14]$ below.

[13] M. Muniruzzmanm, Ph.D. thesis (2003), http://www.phenix.bnl.gov/WWW/talk/theses.php

[14] Deepali Sharma, PhD. thesis (2010), http://www.phenix.bnl.gov/WWW/talk/theses.php .

[15] M. Hirai, S. Kumano, T.-H. Nagai, K. Sudoh, hep-ph/0702250v2, 2007.

[16] J. Binnewies, hep-ph/9707269, 1997.

[17] C. Amsler et al., Phy. Lett. B 667, 1 (2008).

[18] F. Halzen and A. D. Martin, Quarks and Leptons: An introductory course in modern particle physics, John Wiley \& Sons (1984).

[19] J.F. Owens, E. Reya, M. Glück, Phys. Rev. D 18, 1501 (1978).

[20] J.F. Owens and J. D. Kimel, Phys. Rev. D 18, 3313, (1978).

[21] S.M. Beran, J.D. Bjorken, and J. B. Kogut, Phys. Rev. D 4, 3388, (1971).

[22] B.L. Combridge and C.J. Maxwell, Nucl. Phys. B 239, 429 (1984).

[23] K. Nakamura et al. (Particle Data Group), J. Phys. G 37, 075021 (2010).

[24] W.-M. Yao et al., J. Phys. G 33, 1 (2006).

[25] G. D. Lafferty, P. I. Reeves and M. R. Whalley, J. Nucl. Part. Phys. G 21, A1-A151 (1995) contains a compilation of inclusive particle production data in $e^{+} e^{-}$process. Individual data for $\rho, K^{*}, \omega$ and $\phi$ at $Z$ pole are listed separately.

[26] D. Buskulic et al., ALEPH Collab., Z. Phys. C 69, 379 (1996) for the $\rho$ data on the Z-pole.

[27] R. Barate et al., ALEPH Collab., Phys. Rep. 294, 1 (1998) for the $\rho$ data on the $Z$-pole.

[28] P. Abreu et al., DELPHI Collab., Z. Phys. C 65, 587 (1995) for the $\rho$ data on the $Z$-pole.

[29] S. Abachi et al., HRS Collab., Phys. Rev. D 40, 706 (1989) for the low energy $\rho$ data.

[30] D. Buskulic et al., ALEPH Collab., Z. Phys. C 69, 379 (1995) for the inclusive $K^{*}$ data. The "uds" data are listed below[40, 41].

[31] R. Barate et al., ALEPH Collab., Phys. Rep. 294, 1 (1998) for the inclusive $K^{*}$ data. The "uds" data are listed below[40, 41].

[32] P. Abreu et al., DELPHI Collab., Phys. Lett. B 298, 236 (1993) for the inclusive $K^{*}$ data. The "uds" data are listed below[40, 41].

[33] R. Akers et al., OPAL Collab., Z. Phys. C 68, 1 (1995) for the inclusive $K^{*}$ data. The "uds" 
data are listed below $[40,41]$.

[34] D. Buskulic et al., ALEPH Collab., Z. Phys. C 69, 379 (1996) for the $\omega$ data.

[35] R. Barate et al., ALEPH Collab., Phys. Rep. 294, 1 (1996) for the $\omega$ data.

[36] D. Buskulic et al., ALEPH Collab., Z. Phys. C 69, 379 (1996) for the inclusive $\phi$ data. The "uds" data are listed below $[40,41]$.

[37] R. Barate et al., ALEPH Collab., Phys. Rep. 294, 1 (1998) for the inclusive $\phi$ data. The "uds" data are listed below $[40,41]$.

[38] P. Abreu et al., DELPHI Collab., Z. Phys. C 73, 61 (1996) for the inclusive $\phi$ data. The "uds" data are listed below $[40,41]$.

[39] R. Akers et al., OPAL Collab., Z. Phys. C 68, 1 (1995) for the inclusive $\phi$ data. The "uds" data are listed below $[40,41]$.

[40] K. Abe et al., Phys. Rev. D 59, 052001 (1999).

[41] K. Abe et al., Phys. Rev. D 69, 072003 (2003).

[42] M. Glück, E. Reya and A. Vogt, Eur. Phys. J. C 5, 461 (1998), arXiv:hep-ph/9806404. 
TABLE I: Quark fragmentation functions into members of meson octet in terms of the $\mathrm{SU}(3)$ functions, $\alpha, \beta$ and $\gamma$.

\begin{tabular}{|c|c|c|c|}
\hline $\begin{array}{c}\text { fragmenting } \\
\text { quark }\end{array}$ & $K^{*+}$ & $\begin{array}{c}\text { fragmenting } \\
\text { quark }\end{array}$ & $K^{* 0}$ \\
\hline$u$ & $: \alpha+\beta+\frac{3}{4} \gamma$ & $u$ & $: 2 \beta+\gamma$ \\
\hline$d$ & $: 2 \beta+\gamma$ & $d$ & $: \alpha+\beta+\frac{3}{4} \gamma$ \\
\hline$s$ & $: 2 \gamma$ & $s$ & $: 2 \gamma$ \\
\hline $\begin{array}{l}\text { fragmenting } \\
\text { quark }\end{array}$ & $\omega / \phi$ & $\begin{array}{c}\text { fragmenting } \\
\text { quark }\end{array}$ & $\rho^{0}$ \\
\hline$u$ & $: \frac{1}{6} \alpha+\frac{9}{6} \beta+\frac{9}{8} \gamma$ & $u$ & $: \frac{1}{2} \alpha+\frac{1}{2} \beta+\frac{11}{8} \gamma$ \\
\hline$d$ & $: \frac{1}{6} \alpha+\frac{9}{6} \beta+\frac{9}{8} \gamma$ & $d$ & $: \frac{1}{2} \alpha+\frac{1}{2} \beta+\frac{11}{8} \gamma$ \\
\hline$s$ & $: \frac{4}{6} \alpha+\frac{9}{6} \gamma$ & $s$ & $: 2 \beta+\gamma$ \\
\hline $\begin{array}{l}\text { fragmenting } \\
\text { quark }\end{array}$ & $\rho^{+}$ & $\begin{array}{c}\text { fragmenting } \\
\text { quark }\end{array}$ & $\rho^{-}$ \\
\hline$u$ & $: \alpha+\beta+\frac{3}{4} \gamma$ & $u$ & $: 2 \gamma$ \\
\hline$d$ & $: 2 \gamma$ & $d$ & $: \alpha+\beta+\frac{3}{4} \gamma$ \\
\hline$s$ & $: 2 \beta+\gamma$ & $s$ & $: 2 \beta+\gamma$ \\
\hline $\begin{array}{l}\text { fragmenting } \\
\text { quark }\end{array}$ & $\overline{K^{* 0}}$ & $\begin{array}{c}\text { fragmenting } \\
\text { quark }\end{array}$ & $K^{*-}$ \\
\hline$u$ & $: 2 \beta+\gamma$ & $u$ & $: 2 \gamma$ \\
\hline$d$ & $: 2 \gamma$ & $d$ & $: 2 \beta+\gamma$ \\
\hline$s$ & $: \alpha+\beta+\frac{3}{4} \gamma$ & $s$ & $: \alpha+\beta+\frac{3}{4} \gamma$ \\
\hline
\end{tabular}


TABLE II: Best fit values of the parameters defining the input fragmentation functions at the starting scale of $Q^{2}=1.5 \mathrm{GeV}^{2}$, with their 1- $\sigma$ error bars.

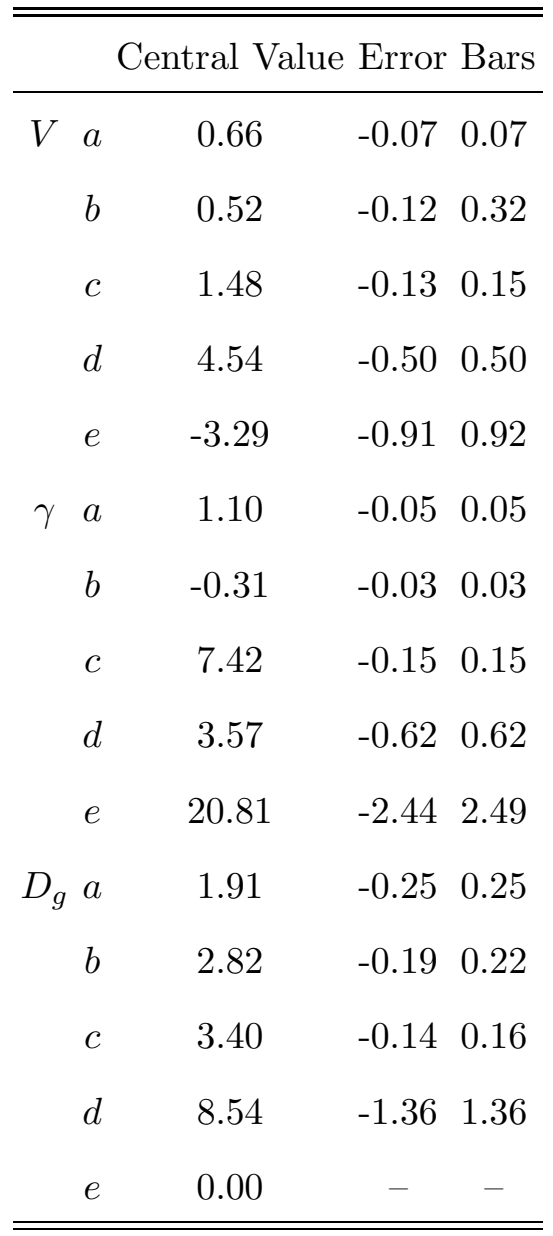

TABLE III: Best fit values of the parameters defining the input fragmentation functions at the starting scale of $Q^{2}=1.5 \mathrm{GeV}^{2}$, with their $1-\sigma$ error bars.

\begin{tabular}{cccc}
\hline \hline \multicolumn{4}{c}{ Central Value Error Bars } \\
\hline$\lambda$ & 0.063 & -0.01 & 0.01 \\
$\theta$ & 42.6 & -2.0 & 2.0 \\
$f_{\text {sea }}^{\omega}$ & 0.94 & -0.08 & 0.08 \\
$f_{1}^{u}(\omega)$ & 0.1 & -0.3 & 0.3 \\
$f_{1}^{s}(\phi)$ & 4.0 & -1.4 & 1.5 \\
$f_{g}^{K^{*}}$ & 1.0 & -0.2 & 0.2 \\
$f_{g}^{\omega}$ & 1.0 & -0.7 & 0.7 \\
$f_{g}^{\phi}$ & 0.32 & -0.08 & 0.09 \\
\hline \hline
\end{tabular}


TABLE IV: $\chi^{2}$ for fits to inclusive vector meson production data from $e^{+} e^{-}$experiments on the $Z$-pole from LEP and SLD experiments and from $p p$ experiments from PHENIX at RHIC.

\begin{tabular}{ccc}
\hline \hline Data Set & No. of data points & $\chi^{2}$ \\
\hline$\rho$ (ALEPH) & 8 & 4.8 \\
$\rho$ (DELPHI '95) & 6 & 2.0 \\
$K^{* 0}$ (SLD) & 6 & 7.1 \\
$\omega($ ALEPH) & 6 & 0.7 \\
$\phi($ SLD $)$ & 6 & 1.0 \\
\hline \hline$\omega($ PHENIX) & 19 & 21.4 \\
$\phi($ PHENIX) & 5 & 5.3 \\
\hline \hline
\end{tabular}

